\title{
DISSEMINATED VILONODULAR SYNOVITIS: CASE REPORT
}

\author{
Gabriela Almeida Barbosa ${ }^{1, \star}$, Robson Antônio Gonçalves ${ }^{1}$, Valéria Bezerra da Silva ${ }^{1}$, Tâmara Santos Melo ${ }^{1}$, Alessandra de \\ Sousa Braz ${ }^{1}$
}

1.Universidade Federal da Paraíba, João Pessoa (PB), Brazil.

*Corresponding author: gabrielaalmeidamed@gmail.com

\section{BACKGROUND}

Pigmented villonodular synovitis (PVNS) is a rare benign proliferative lesion of unknown cause, more frequent in the third and fourth decades of life. It is oligoarticular in most cases, the knee being the most affected joint. Polyarticular/disseminated involvement of PVNS is uncommon, with difficult diagnosis and management.

\section{CASE REPORT}

Female, 46 years old, with severe pain in her left knee, associated with increased volume and temperature, after trauma, for 23 years. She started treatment with nonsteroidal anti-inflammatory drugs (NSAIDs) with partial resolution of symptoms. Magnetic resonance imaging (MRI) of the left knee showed a lesion in the medial retinaculum of the patella and a small intra-articular effusion with a reduction in the medial femorotibial space on radiography. Triamcinolone infiltration was performed, and symptoms improved. In 2002, she developed erythematous macules on the left knee, with normal platelet count and coagulogram, in addition to low back pain with mechanical characteristics. Two years later, she had persistent low back pain and arthritis in her left knee and progressed to arthritis in her right knee. Intra-articular puncture was performed with the presence of serohemorrhagic synovial fluid (SF), subjected to culture for bacteria, fungi and Koch's bacillus, without growth. Progression of the investigation with synovial biopsy showing histopathological findings compatible with PVNS. Thirteen years ago, she developed wrist arthritis, which improved after the introduction of glucosamine sulfate and paracetamol, remaining asymptomatic in subsequent years. In 2016, she returned with knee arthritis, limited movement, with a new puncture showing serohemorrhagic SF and a new histopathological finding compatible with PVNS. Hydroxychloroquine and colchicine were prescribed, with no improvement, and later methotrexate, with low adherence. The patient evolved one year ago with unintentional weight loss $(15 \mathrm{~kg})$, progressive and disabling arthritis of the knees, ankles, feet and hips, and periarticular ecchymosis (Figure 1). Magnetic resonance imaging showing signs of synovial hypertrophy with postcontrast enhancement in knees and hips. Tofacitinib was started with a report of worsening pain and currently using prednisone $20 \mathrm{mg} /$ day and naproxen $1 \mathrm{~g} /$ day, with partial relief; however, in bed rest.

(a)

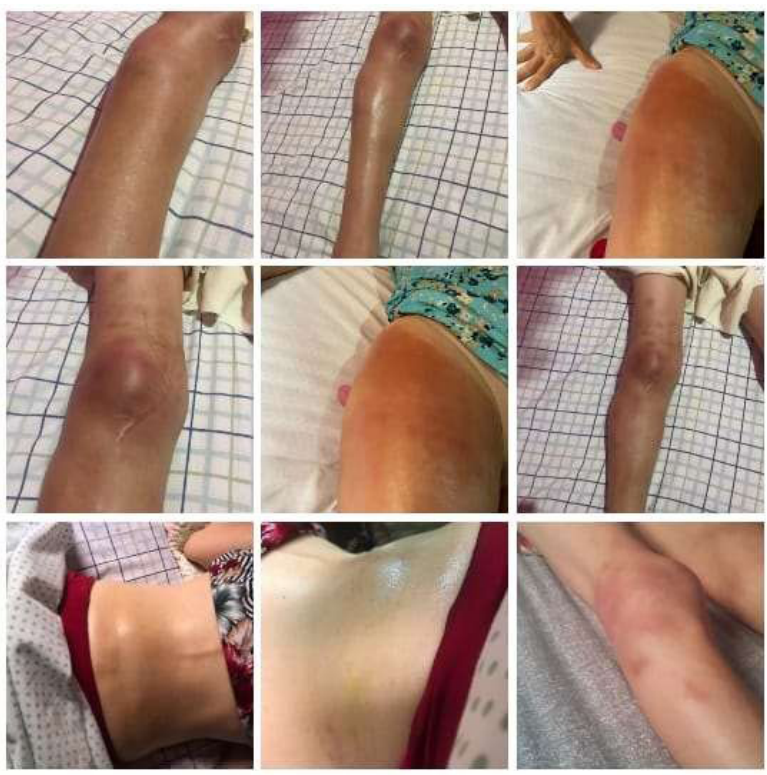

(b)

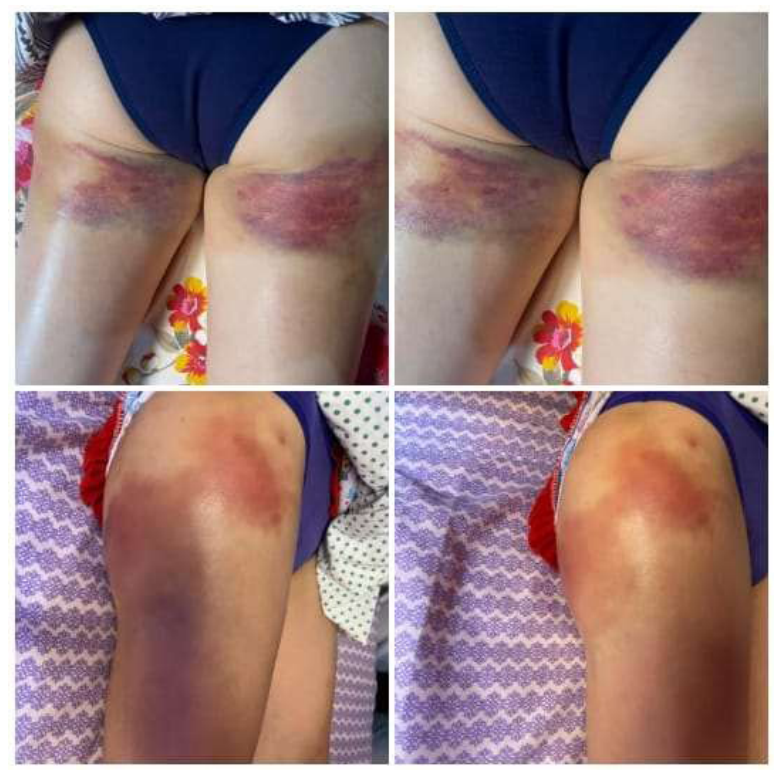

Figure 1. Periarticular ecchymosis in a patient with a previous diagnosis of PVNS.

Realização: 


\section{CONCLUSION}

Pigmented villonodular synovitis is a difficult disease to diagnose. The disseminated form is noteworthy for its rarity, with few case reports described in the literature. Periarticular involvement in the form of tenosynovitis, enthesitis and bursitis may be present and contribute to limited movement. Therapeutic management remains challenging, with little literary evidence.

\section{KEYWORDS}

Pigmented villonodular synovitis, Polyarticular, Magnetic resonance. 